\title{
The existence of fixed points for new nonlinear multivalued maps and their applications
}

Zhenhua He${ }^{1}$, Wei-Shih $\mathrm{Du}^{2^{*}}$ and Ing-Jer Lin ${ }^{2}$

\author{
* Correspondence: wsdu@nknucc. \\ nknu.edu.tw \\ ${ }^{2}$ Department of Mathematics, \\ National Kaohsiung Normal \\ University, Kaohsiung 824, Taiwan \\ Full list of author information is \\ available at the end of the article
}

\section{Abstract}

In this paper, we first establish some new fixed point theorems for $\mathcal{M T}$-functions. By using these results, we can obtain some generalizations of Kannan's fixed point theorem and Chatterjea's fixed point theorem for nonlinear multivalued contractive maps in complete metric spaces. Our results generalize and improve some main results in the literature and references therein.

\section{Mathematics Subject Classifications}

47H10; $54 \mathrm{H} 25$

Keywords: $\tau$-function, MT-function, function of contractive factor, Kannan's fixed point theorem, Chatterjea's fixed point theorem

\section{Introduction}

Throughout this paper, we denote by $\mathbb{N}$ and $\mathbb{R}$, the sets of positive integers and real numbers, respectively. Let $(X, d)$ be a metric space. For each $x \in X$ and $A \subseteq X$, let $d(x$, $A)=\inf _{y \in A} d(x, y)$. Denote by $\mathcal{N}(X)$ the family of all nonempty subsets of $X, \mathcal{C}(X)$ the family of all nonempty closed subsets of $X$ and $\mathcal{C B}(X)$ the class of all nonempty closed bounded subsets of $X$, respectively.

For any $A, B \in \mathcal{C B}(X)$, define a function $\mathcal{H}: \mathcal{C B}(X) \times \mathcal{C B}(X) \rightarrow[0, \infty)$ by

$$
\mathcal{H}(A, B)=\max \left\{\sup _{x \in B} d(x, A), \sup _{x \in A} d(x, B)\right\},
$$

then $\mathcal{H}$ is said to be the Hausdorff metric on $\mathcal{C B}(X)$ induced by the metric $d$ on $X$. A point $x$ in $X$ is a fixed point of a map $T$ if $T x=x$ (when $T: X \rightarrow X$ is a single-valued map) or $x \in T x$ (when $T: X \rightarrow 2^{X}$ is a multivalued map). The set of fixed points of $T$ is denoted by $\mathcal{F}(T)$.

It is known that many metric fixed point theorems were motivated from the Banach contraction principle (see, e.g., [1]) that plays an important role in various fields of applied mathematical analysis. Later, Kannan [2,3] and Chatterjea [4] established the following fixed point theorems.

Theorem K. (Kannan [2,3]) Let $(X, d)$ be a complete metric space and $T: X \rightarrow X$ be a selfmap. Suppose that there exists $\gamma \in\left[0, \frac{1}{2}\right)$ such that

$$
d(T x, T y) \leq \gamma(d(x, T x)+d(y, T y)) \text { for all } x, y \in X .
$$

Then, $T$ has a unique fixed point in $X$. 
Theorem C. (Chatterjea [4]) Let $(X, d)$ be a complete metric space and $T: X \rightarrow X$ be a selfmap. Suppose that there exists $\gamma \in\left[0, \frac{1}{2}\right)$ such that

$$
d(T x, T y) \leq \gamma(d(x, T y)+d(y, T x)) \text { for all } x, y \in X .
$$

Then, $T$ has a unique fixed point in $X$.

Let $f$ be a real-valued function defined on $\mathbb{R}$. For $c \in \mathbb{R}$, we recall that

$$
\limsup _{x \rightarrow c} f(x)=\inf _{\varepsilon>0} \sup _{0<|x-c|<\varepsilon} f(x)
$$

and

$$
\limsup _{x \rightarrow c^{+}} f(x)=\inf _{\varepsilon>0} \sup _{0<x-c<\varepsilon} f(x) .
$$

Definition 1.1. [5-10] A function $\phi:[0, \infty) \rightarrow[0,1)$ is said to be an $\mathcal{M T}$-function if it satisfies Mizoguchi-Takahashi's condition (i.e., $\lim \sup _{s} \rightarrow t^{+} \phi(s)<1$ for all $t \in[0, \infty)$ ).

It is obvious that if $\phi:[0, \infty) \rightarrow[0,1)$ is a nondecreasing function or a nonincreasing function, then $\phi$ is an $\mathcal{M T}$-function. So the set of $\mathcal{M T}$-functions is a rich class. But it is worth to mention that there exist functions that are not $\mathcal{M T}$-functions.

Example 1.1. [8] Let $\phi:[0, \infty) \rightarrow[0,1)$ be defined by

$$
\varphi(t):=\left\{\begin{array}{cc}
\frac{\sin t}{t}, & \text { if } t \in\left(0, \frac{\pi}{2}\right] \\
0, & \text { otherwise }
\end{array}\right.
$$

Since $\lim \sup _{s \rightarrow 0^{+}} \varphi(s)=1, \varphi$ is not an $\mathcal{M T}$-function.

Very recently, $\mathrm{Du}[8]$ first proved some characterizations of $\mathcal{M T}$-functions.

Theorem D. [8] Let $\phi:[0, \infty) \rightarrow[0,1)$ be a function. Then, the following statements are equivalent.

(a) $\phi$ is an $\mathcal{M T}$-function.

(b) For each $t \in[0, \infty)$, there exist $r_{t}^{(1)} \in[0,1)$ and $\varepsilon_{t}^{(1)}>0$ such that $\varphi(s) \leq r_{t}^{(1)}$ for all $s \in\left(t, t+\varepsilon_{t}^{(1)}\right)$.

(c) For each $t \in[0, \infty)$, there exist $r_{t}^{(2)} \in[0,1)$ and $\varepsilon_{t}^{(2)}>0$ such that $\varphi(s) \leq r_{t}^{(2)}$ for all $s \in\left[t, t+\varepsilon_{t}^{(2)}\right]$.

(d) For each $t \in[0, \infty)$, there exist $r_{t}^{(3)} \in[0,1)$ and $\varepsilon_{t}^{(3)}>0$ such that $\varphi(s) \leq r_{t}^{(3)}$ for all $s \in\left(t, t+\varepsilon_{t}^{(3)}\right]$.

(e) For each $t \in[0, \infty)$, there exist $r_{t}^{(4)} \in[0,1)$ and $\varepsilon_{t}^{(4)}>0$ such that $\varphi(s) \leq r_{t}^{(4)}$ for all $s \in\left[t, t+\varepsilon_{t}^{(4)}\right)$.

(f) For any nonincreasing sequence $\left\{x_{n}\right\}_{n \in \mathbb{N}}$ in $[0, \infty)$, we have $0 \leq \sup _{n \in \mathbb{N}} \phi\left(x_{n}\right)<1$.

(g) $\phi$ is a function of contractive factor [10]; that is, for any strictly decreasing sequence $\left\{x_{n}\right\}_{n \in \mathbb{N}}$ in $[0, \infty)$, we have $0 \leq \sup _{n \in \mathbb{N}} \phi\left(x_{n}\right)<1$.

In 2007, Berinde and Berinde [11] proved the following interesting fixed point theorem.

Theorem BB. (Berinde and Berinde [11]) Let $(X, d)$ be a complete metric space, $T: X \rightarrow \mathcal{C B}(X)$ be a multivalued map, $\phi:[0, \infty) \rightarrow[0,1)$ be an $\mathcal{M T}$-function and $L \geq$ 
0. Assume that

$$
\mathcal{H}(T x, T y) \leq \varphi(d(x, y)) d(x, y)+L d(y, T x) \quad \text { for all } x, y \in X
$$

Then $\mathcal{F}(T) \neq \varnothing$.

It is quite obvious that if let $L=0$ in Theorem BB, then we can obtain MizoguchiTakahashi's fixed point theorem [12] that is a partial answer of Problem 9 in Reich $[13,14]$.

Theorem MT. (Mizoguchi and Takahashi [12]) Let $(X, d)$ be a complete metric space, $T: X \rightarrow \mathcal{C B}(X)$ be a multivalued map and $\phi:[0, \infty) \rightarrow[0,1)$ be an $\mathcal{M T}$-function. Assume that

$$
\mathcal{H}(T x, T y) \leq \varphi(d(x, y)) d(x, y) \quad \text { for all } x, y \in X
$$

Then $\mathcal{F}(T) \neq \emptyset$.

In fact, Mizoguchi-Takahashi's fixed point theorem is a generalization of Nadler's fixed point theorem, but its primitive proof is difficult. Later, Suzuki [15] give a very simple proof of Theorem MT. Recently, Du [5] established new fixed point theorems for $\tau^{0}$-metric (see Def. 2.1 below) and $\mathcal{M T}$-functions to extend Berinde-Berinde's fixed point theorem. In [5], some generalizations of Kannan's fixed point theorem, Chatterjea's fixed point theorem and other new fixed point theorems for nonlinear multivalued contractive maps were given.

In this paper, we first establish some new fixed point theorems for $\mathcal{M T}$-functions. By using these results, we can obtain some generalizations of Kannan's fixed point theorem and Chatterjea's fixed point theorem for nonlinear multivalued contractive maps in complete metric spaces. Our results generalize and improve some main results in [1-5,7-9,12-15] and references therein.

\section{Preliminaries}

Let $(X, d)$ be a metric space. Recall that a function $p: X \times X \rightarrow[0, \infty)$ is called a $w$-distance $[1,16,17]$, if the following are satisfied:

$(w 1) p(x, z) \leq p(x, y)+p(y, z)$ for any $x, y, z \in X$;

$(w 2)$ for any $x \in X, p(x, \cdot): X \rightarrow[0, \infty)$ is l.s.c;

(w3) for any $\varepsilon>0$, there exists $\delta>0$ such that $p(z, x) \leq \delta$ and $p(z, y) \leq \delta$ imply $d(x, y)$ $\leq \varepsilon$.

Recently, Lin and Du introduced and studied $\tau$-functions [5,9,18-22]. A function $p: X$ $\times X \rightarrow[0, \infty)$ is said to be a $\tau$-function, if the following conditions hold:

$(\tau 1) p(x, z) \leq p(x, y)+p(y, z)$ for all $x, y, z \in X$;

$(\tau 2)$ If $x \in X$ and $\left\{y_{n}\right\}$ in $X$ with $\lim _{n \rightarrow \infty} y_{n}=y$ such that $p\left(x, y_{n}\right) \leq M$ for some $M=$ $M(x)>0$, then $p(x, y) \leq M$;

$(\tau 3)$ For any sequence $\left\{x_{n}\right\}$ in $X$ with $\lim _{n \rightarrow \infty} \sup \left\{p\left(x_{n}, x_{m}\right): m>n\right\}=0$, if there exists a sequence $\left\{y_{n}\right\}$ in $X$ such that $\lim _{n \rightarrow \infty} p\left(x_{n}, y_{n}\right)=0$, then $\lim _{n \rightarrow \infty} d\left(x_{n}, y_{n}\right)=0$;

$(\tau 4)$ For $x, y, z \in X, p(x, y)=0$ and $p(x, z)=0$ imply $y=z$.

Note that not either of the implications $p(x, y)=0 \Leftrightarrow x=y$ necessarily holds and $p$ is nonsymmetric in general. It is well-known that the metric $d$ is a $w$-distance and any $w$ distance is a $\tau$-function, but the converse is not true; see $[5,19]$.

The following Lemma is essentially proved in [19]. See also $[5,8,20,22]$. 
Lemma 2.1. $[5,8,19,20,22]$ Let $(X, d)$ be a metric space and $p: X \times X \rightarrow[0, \infty)$ be any function. Then, the following hold:

(a) If $p$ satisfies ( $w 2)$, then $p$ satisfies $(\tau 2)$;

(b) If $p$ satisfies $(w 1)$ and ( $w 3)$, then $p$ satisfies ( $\tau 3)$;

(c) Assume that $p$ satisfies ( $\tau 3)$. If $\left\{x_{n}\right\}$ is a sequence in $X$ with $\lim _{n \rightarrow \infty} \sup \left\{p\left(x_{n}\right.\right.$, $\left.\left.x_{m}\right): m>n\right\}=0$, then $\left\{x_{n}\right\}$ is a Cauchy sequence in $X$.

Let $(X, d)$ be a metric space and $p: X \times X \rightarrow[0, \infty)$ a $\tau$-function. For each $x \in X$ and $\mathrm{A} \subseteq \mathrm{X}$, let

$$
d(x, A)=\inf _{y \in A} d(x, y) .
$$

Recall that a selfmap $T: X \rightarrow X$ is said to be

(a) Kannan's type $[2,5,16]$ if there exists $\gamma \in\left[0, \frac{1}{2}\right)$, such that $d(T x, T y) \leq \gamma\{d(x$, $T x)+d(y, T y)\}$ for all $x, y \in X$;

(b) Chatterjea's type [3,5] if there exists $\gamma \in\left[0, \frac{1}{2}\right)$, such that $d(T x, T y) \leq \gamma\{d(x, T y)$

$+d(y, T x)\}$ for all $x, y \in X$.

Lemma 2.2. $[5,9,21,22]$ Let $A$ be a closed subset of a metric space $(X, d)$ and $p: X \times$ $X \rightarrow[0, \infty)$ be any function. Suppose that $p$ satisfies $(\tau 3)$ and there exists $u \in X$ such that $p(u, u)=0$. Then, $p(u, A)=0$ if and only if $u \in A$.

Recently, Du [5,21] first has introduced the concepts of $\tau^{0}$-functions and $\tau^{0}$-metrics as follows.

Definition 2.1. $[5,9,21,22]$ Let $(X, d)$ be a metric space. A function $p: X \times X \rightarrow[0$, $\infty)$ is called a $\tau^{0}$-function if it is a $\tau$-function on $X$ with $p(x, x)=0$ for all $x \in X$.

Remark 2.1. If $p$ is a $\tau^{0}$-function then, from $(\tau 4), p(x, y)=0$ if and only if $x=y$.

Example 2.1. [5] Let $X=\mathbb{R}$ with the metric $d(x, y)=|x-y|$ and $0<a<b$. Define the function $p: X \times X \rightarrow[0, \infty)$ by

$$
p(x, y)=\max \{a(y-x), b(x-y)\} .
$$

Then, $p$ is nonsymmetric, and hence, $p$ is not a metric. It is easy to see that $p$ is a $\tau^{0}$ function.

Definition 2.2. $[5,9,21,22]$ Let $(X, d)$ be a metric space and $p$ be a $\tau^{0}$-function (resp. $w^{0}$-distance). For any $A, B \in \mathcal{C B}(X)$, define a function $\mathcal{D}_{p}: \mathcal{C B}(X) \times \mathcal{C B}(X) \rightarrow[0, \infty)$ by

$$
\mathcal{D}_{p}(A, B)=\max \left\{\delta_{p}(A, B), \delta_{p}(B, A)\right\},
$$

where $\delta_{p}(A, B)=\sup _{x \in A} p(x, B)$ and $\delta_{p}(B, A)=\sup _{x \in B} p(x, A)$, then $\mathcal{D}_{p}$ is said to be the $\tau^{0}$-metric (resp. $w^{0}$-metric) on $\mathcal{C B}(X)$ induced by $p$.

Clearly, any Hausdorff metric is a $\tau^{0}$-metric, but the reverse is not true. It is wellknown that every $\tau^{0}$-metric $\mathcal{D}_{p}$ is a metric on $\mathcal{C B}(X)$; for more detail, see $[5,9,21,22]$.

Lemma 2.3. Let $(X, d)$ be a metric space, $T: X \rightarrow \mathcal{C}(X)$ be a multivalued map and $\left\{z_{n}\right\}$ be a sequence in $X$ satisfying $z_{n+1} \in T z_{n}, n \in \mathbb{N}$, and $\left\{z_{n}\right\}$ converge to $v$ in $X$. 
Then, the following statements hold.

(a) If $T$ is closed (that is, $\operatorname{Gr} T=\{(x, y) \in X \times X: y \in T x\}$, the graph of $T$, is closed in $X \times X)$, then $\mathcal{F}(T) \neq \emptyset$.

(b) Let $p$ be a function satisfying $(\tau 3)$ and $p(v, v)=0$. If $\lim _{n \rightarrow \infty} p\left(z_{n}, z_{n+1}\right)=0$ and the map $f: X \rightarrow[0, \infty)$ defined by $f(x)=p(x, T x)$ is l.s.c., then $\mathcal{F}(T) \neq \emptyset$.

(c) If the map $g: X \rightarrow[0, \infty)$ defined by $g(x)=d(x, T x)$ is l.s.c., then $\mathcal{F}(T) \neq \emptyset$.

(d) Let $p$ be a function satisfying ( $\tau 3)$. If $\lim _{n \rightarrow \infty} p\left(z_{n}, T v\right)=0$ and $\lim _{n \rightarrow \infty} \sup \{p$ $\left.\left(z_{n}, z_{m}\right): m>n\right\}=0$, then $\mathcal{F}(T) \neq \emptyset$.

\section{Proof.}

(a) Since $T$ is closed, $z_{n+1} \in T z_{n}, n \in \mathbb{N}$ and $z_{n} \rightarrow v$ as $n \rightarrow \infty$, we have $v \in T v$. So $\mathcal{F}(T) \neq \emptyset$.

(b) Since $z_{n} \rightarrow v$ as $n \rightarrow \infty$, by the lower semicontinuity of $f$, we obtain

$$
p(v, T v)=f(v) \leq \liminf _{m \rightarrow \infty} p\left(z_{n}, T z_{n}\right) \leq \lim _{n \rightarrow \infty} p\left(z_{n}, z_{n+1}\right)=0,
$$

which implies $p(v, T v)=0$. By Lemma 2.2, we get $v \in \mathcal{F}(T)$.

(c) Since $\left\{z_{n}\right\}$ is convergent in $X, \lim _{n \rightarrow \infty} d\left(z_{n}, z_{n}+1\right)=0$. Since

$$
d(v, T v)=g(v) \leq \liminf _{m \rightarrow \infty} d\left(z_{n}, T z_{n}\right) \leq \lim _{n \rightarrow \infty} d\left(z_{n}, z_{n+1}\right)=0,
$$

we have $d(v, T v)=0$ and hence $v \in \mathcal{F}(T)$.

(d) Since $\lim _{n \rightarrow \infty} \sup \left\{p\left(z_{n}, z_{m}\right): m>n\right\}=0$ and $\lim _{n \rightarrow \infty} p\left(z_{n}, T v\right)=0$, there exists $\left\{a_{n}\right\} \subset\left\{z_{n}\right\}$ with $\lim _{n \rightarrow \infty} \sup \left\{\mathrm{p}\left(a_{n}, a_{m}\right): m>n\right\}=0$ and $\left\{b_{n}\right\} \subset T v$ such that $\lim _{n \rightarrow \infty}$ $p\left(a_{n}, b_{n}\right)=0$. By $(\tau 3), \lim _{n \rightarrow \infty} d\left(a_{n}, b_{n}\right)=0$. Since $a_{n} \rightarrow v$ as $n \rightarrow \infty$ and $d\left(b_{n}, v\right) \leq$ $d\left(b_{n}, a_{n}\right)+d\left(a_{n}, v\right)$, it implies $b_{n} \rightarrow v$ as $n \rightarrow \infty$. By the closedness of $T v$, we have $v$

$\in T v$ or $v \in \mathcal{F}(T)$.

In this paper, we first introduce the concepts of capable maps as follows.

Definition 2.3. Let $(X, d)$ be a metric space and $T: X \rightarrow \mathcal{C}(X)$ be a multivalued map. We say that $T$ is capable if $T$ satisfies one of the following conditions:

(D1) $T$ is closed;

(D2) the map $f: X \rightarrow[0, \infty)$ defined by $f(x)=p(x, T x)$ is l.s.c;

(D3) the map $g: X \rightarrow[0, \infty)$ defined by $g(x)=d(x, T x)$ is l.s.c;

(D4) for each sequence $\left\{x_{n}\right\}$ in $X$ with $x_{n+1} \in T x_{n}, n \in \mathbb{N}$ and $\lim _{n \rightarrow \infty} x_{n}=v$, we have $\lim _{n \rightarrow \infty} p\left(x_{n}, T v\right)=0$;

(D5) $\inf \{p(x, z)+p(x, T x): x \in X\}>0$ for every $z \notin \mathcal{F}(T)$.

\section{Remark 2.2.}

(1) Let $(X,\|\cdot\|)$ be a Banach space. If $T: X \rightarrow \mathcal{C}(X)$ is u.s.c, then $T$ is a capable map since it is closed (for more detail, see $[5,23]$ ). 
(2) Let $(X, d)$ be a metric space and $T: X \rightarrow \mathcal{C}(X)$ be u.s.c. Since the function $f: X$ $\rightarrow[0, \infty)$ defined by $f(x)=d(x, T x)$ is 1.s.c. (see, e.g., [24, Lemma 3.1] and [25, Lemma 2]), $T$ is a capable map.

(3) Let $(X, d)$ be a metric space and $T: X \rightarrow \mathcal{C B}(X)$ be a generalized multivalued $(\phi, L)$-weak contraction [11], that is, there exists an $\mathcal{M T}$-function $\phi$ and $L \geq 0$ such that

$$
\mathcal{H}(T x, T y) \leq \varphi(d(x, y)) d(x, y)+L d(y, T x) \quad \text { for all } x, y \in X
$$

Then, $T$ is a capable map. Indeed, let $\left\{x_{n}\right\}$ in $X$ with $x_{n+1} \in T x_{n}, n \in \mathbb{N}$ and $\lim _{n \rightarrow \infty}$ $x_{n}=v$.

Then

$$
\begin{aligned}
\lim _{n \rightarrow \infty} d\left(x_{n+1}, T v\right) & \leq \lim _{n \rightarrow \infty} \mathcal{H}\left(T x_{n}, T v\right) \\
& \leq \lim _{n \rightarrow \infty}\left\{\varphi\left(d\left(x_{n}, v\right)\right) d\left(x_{n}, v\right)+L d\left(v, x_{n+1}\right)\right\}=0
\end{aligned}
$$

which means that $T$ satisfies (D4).

(4) Let $(X, d)$ be a metric space and $T: X \rightarrow X$ is a single-valued map of Kannan's type, then $T$ is a capable map since (D5) holds; for more detail, see [[16], Corollary 3].

\section{Fixed point theorems of generalized Chatterjea's type and others}

Below, unless otherwise specified, let $(X, d)$ be a complete metric space, $p$ be a $\tau^{0}$-function and $\mathcal{D}_{p}$ be a $\tau^{0}$-metric on $\mathcal{C B}(X)$ induced by $p$.

In this section, we will establish some fixed point theorems of generalized Chatterjea's type.

Theorem 3.1. Let $T: X \rightarrow \mathcal{C}(X)$ be a capable map. Suppose that there exists an $\mathcal{M T}$-function $\phi:[0, \infty) \rightarrow[0,1)$ such that for each $x \in X$,

$$
2 p(y, T y) \leq \varphi(p(x, y)) p(x, T y) \quad \text { for all } y \in T x .
$$

Then $\mathcal{F}(T) \neq \emptyset$.

Proof. Let $\kappa:[0, \infty) \rightarrow[0,1)$ be defined by $\kappa(t)=\frac{1+\varphi(t)}{2}$. Then

$$
0 \leq \varphi(t)<\kappa(t)<1 \quad \text { for all } t \in[0, \infty)
$$

Let $x_{1} \in X$ and $x_{2} \in T x_{1}$. If $x_{1}=x_{2}$, then $x_{1} \in \mathcal{F}(T)$ and we are done. Otherwise, if $x_{2} \neq x_{1}$, by Remark 2.1, we have $p\left(x_{1}, x_{2}\right)>0$. If $x_{1} \in T x_{2}$, then it follows from (3.1) that

$$
2 p\left(x_{2}, T x_{2}\right) \leq \varphi\left(p\left(x_{1}, x_{2}\right)\right) p\left(x_{1}, T x_{2}\right)=0,
$$

which implies $p\left(x_{2}, T x_{2}\right)=0$. Since $p$ is a $\tau^{0}$-function and $T x_{2}$ is closed in $X$, by Lemma 2.2, $x_{2} \in T x_{2}$ and $x_{2} \in \mathcal{F}(T)$. If $x_{1} \notin T x_{2}$, then $p\left(x_{1}, T x_{2}\right)>0$ and, by (3.1), there exists $x_{3} \in T x_{2}$ such that

$$
\begin{aligned}
2 p\left(x_{2}, x_{3}\right) & <\kappa\left(p\left(x_{1}, x_{2}\right)\right) p\left(x_{1}, x_{3}\right) \\
& \leq \kappa\left(p\left(x_{1}, x_{2}\right)\right)\left[p\left(x_{1}, x_{2}\right)+p\left(x_{2}, x_{3}\right)\right] .
\end{aligned}
$$


By induction, we can obtain a sequence $\left\{x_{n}\right\}$ in $X$ satisfying $x_{n+1} \in T x_{n}, n \in \mathbb{N}, p\left(x_{n}\right.$, $\left.x_{n+1}\right)>0$

and

$$
2 p\left(x_{n+1}, x_{n+2}\right)<\kappa\left(p\left(x_{n}, x_{n+1}\right)\right)\left[p\left(x_{n}, x_{n+1}\right)+p\left(x_{n+1}, x_{n+2}\right)\right]
$$

By (3.2), we get

$$
p\left(x_{n+1}, x_{n+2}\right)<\frac{\kappa\left(p\left(x_{n}, x_{n+1}\right)\right)}{2-\kappa\left(p\left(x_{n}, x_{n+1}\right)\right)} p\left(x_{n}, x_{n+1}\right)
$$

Since $0<\kappa(t)<1$ for all $t \in[0, \infty), \frac{\kappa\left(p\left(x_{n}, x_{n+1}\right)\right)}{2-\kappa\left(p\left(x_{n}, x_{n+1}\right)\right)} \in(0,1)$ for all $n \in \mathbb{N}$. So the sequence $\left\{p\left(x_{n}, x_{n+1}\right)\right\}$ is strictly decreasing in $[0, \infty)$. Since $\phi$ is an $\mathcal{M T}$-function, by applying (g) of Theorem D, we have

$$
0 \leq \sup _{n \in \mathbb{N}} \varphi\left(p\left(x_{n}, x_{n+1}\right)\right)<1 .
$$

Hence, it follows that

$$
0<\sup _{n \in \mathbb{N}} \kappa\left(p\left(x_{n}, x_{n+1}\right)\right)=\frac{1}{2}\left[1+\sup _{n \in \mathbb{N}} \varphi\left(p\left(x_{n}, x_{n+1}\right)\right)\right]<1 .
$$

Let $\lambda:=\sup _{n \in \mathbb{N}} \kappa\left(p\left(x_{n}, x_{n+1}\right)\right)$ and take $c:=\frac{\lambda}{2-\lambda}$. Then $\lambda, c \in(0,1)$. We claim that $\left\{x_{n}\right\}$ is a Cauchy sequence in $X$. Indeed, by (3.3), we have

$$
p\left(x_{n+1}, x_{n+2}\right)<\frac{\kappa\left(p\left(x_{n}, x_{n+1}\right)\right)}{2-\kappa\left(p\left(x_{n}, x_{n+1}\right)\right)} p\left(x_{n}, x_{n+1}\right) \leq c p\left(x_{n}, x_{n+1}\right) \text {. }
$$

It implies from (3.4) that

$$
p\left(x_{n+1}, x_{n+2}\right)<c p\left(x_{n}, x_{n+1}\right)<\cdots<c^{n} p\left(x_{1}, x_{2}\right) \quad \text { for each } n \in \mathbb{N} \text {. }
$$

We have $\lim _{n \rightarrow \infty} \sup \left\{p\left(x_{n}, x_{m}\right): m>n\right\}=0$. Indeed, let $\alpha_{n}=\frac{c^{n-1}}{1-c} p\left(x_{1}, x_{2}\right), n \in \mathbb{Z}$. For $m, n \in \mathbb{N}$ with $m>n$, we have

$$
p\left(x_{n}, x_{m}\right) \leq \sum_{j=n}^{m-1} p\left(x_{j}, x_{j+1}\right)<\alpha_{n} \text {. }
$$

Since $c \in(0,1), \lim _{n \rightarrow \infty} \alpha_{\mathrm{n}}=0$ and, by (3.5), we get

$$
\lim _{n \rightarrow \infty} \sup \left\{p\left(x_{n}, x_{m}\right): m>n\right\}=0 .
$$

Applying (c) of Lemma 2.1, $\left\{x_{n}\right\}$ is a Cauchy sequence in $X$. By the completeness of $X$, there exists $v \in X$ such that $x_{n} \rightarrow v$ as $n \rightarrow \infty$. From $(\tau 2)$ and (3.5), we have

$$
p\left(x_{n}, v\right) \leq \alpha_{n} \quad \text { for all } n \in \mathbb{N} .
$$

Now, we verify that $v \in \mathcal{F}(T)$. Applying Lemma 2.3, we know that $v \in \mathcal{F}(T)$ if $T$ satisfies one of the conditions (D1), (D2), (D3) and (D4).

Finally, assume (D5) holds. On the contrary, suppose that $v \notin T v$. Then, by (3.5) and (3.7), we have 


$$
\begin{aligned}
0 & <\inf _{x \in X}\{p(x, v)+p(x, T x)\} \\
& \leq \inf _{n \in \mathbb{N}}\left\{p\left(x_{n}, v\right)+p\left(x_{n}, T x_{n}\right)\right\} \\
& \leq \inf _{n \in \mathbb{N}}\left\{p\left(x_{n}, v\right)+p\left(x_{n}, x_{n+1}\right)\right\} \\
& \leq \lim _{n \rightarrow \infty} 2 \alpha_{n} \\
& =0,
\end{aligned}
$$

a contradiction. Therefore $v \in \mathcal{F}(T)$. The proof is completed.

Here, we give a simple example illustrating Theorem 3.1.

Example 3.1. Let $X=[0,1]$ with the metric $d(x, y)=|x-y|$ for $x, y \in X$. Then, $(X, d)$ is a complete metric space. Let $T: X \rightarrow \mathcal{C}(X)$ be defined by

$$
T(x)=\left\{\begin{aligned}
\{0,1\}, & \text { if } x=0 \\
\left\{\frac{1}{2} x^{3}, 1\right\}, & \text { if } x \in\left(0, \frac{1}{2}\right] \\
\left\{0, \frac{1}{2} x^{3}\right\}, & \text { if } x \in\left(\frac{1}{2}, 1\right) \\
\{1\}, & \text { if } x=1
\end{aligned}\right.
$$

and $\phi:[0, \infty) \rightarrow[0,1)$ be defined by

$$
\varphi(t)=\left\{\begin{array}{c}
2 t, \text { if } t \in\left[0, \frac{1}{2}\right) \\
0, \text { if } t \in\left[\frac{1}{2}, \infty\right)
\end{array}\right.
$$

Then, $\phi$ is an $\mathcal{M T}$-function and $\mathcal{F}(T)=\{0,1\} \neq 0$.

On the other hand, one can easily see that

$$
d(x, T x)= \begin{cases}x-\frac{1}{2} x^{3}, & \text { if } x \in[0,1) \\ 0, & \text { if } x=1\end{cases}
$$

So $f(x):=d(x, T x)$ is l.s.c., and hence, $T$ is a capable map. Moreover, it is not hard to verify that for each $x \in X$,

$$
2 p(y, T y) \leq \varphi(p(x, y)) p(x, T y) \quad \text { for all } y \in T x
$$

Therefore, all the assumptions of Theorem 3.1 are satisfied, and we also show that $\mathcal{F}(T) \neq \emptyset$ from Theorem 3.1 .

Theorem 3.2. Let $T: X \rightarrow \mathcal{C}(X)$ be a capable map and $\phi:[0, \infty) \rightarrow[0,1)$ be an $\mathcal{M T}$-function. Let $k \in \mathbb{R}$ with $k \geq 2$. Suppose that for each $x \in X$

$$
k p(y, T y) \leq \varphi(p(x, y)) p(x, T y) \quad \text { for all } y \in T x
$$

Then $\mathcal{F}(T) \neq \emptyset$.

Proof. Since $k \geq 2$, (3.9) implies (3.1). Therefore, the conclusion follows from Theorem 3.1 .

The following result is immediate from the definition of $\mathcal{D}_{p}$ and Theorem 3.1.

Theorem 3.3. Let $T: X \rightarrow \mathcal{C B}(X)$ be a capable map. Suppose that there exists an $\mathcal{M T}$-function $\phi:[0, \infty) \rightarrow[0,1)$ such that for each $x \in X$,

$$
2 \mathcal{D}_{p}(T x, T y) \leq \varphi(p(x, y)) p(x, T y) \quad \text { for all } y \in T x
$$

Then $\mathcal{F}(T) \neq \emptyset$. 
Theorem 3.4. Let $T: X \rightarrow \mathcal{C B}(X)$ be a capable map. Suppose that there exist two $\mathcal{M T}$-functions $\phi, \tau:[0, \infty) \rightarrow[0,1)$ such that

$$
2 \mathcal{D}_{p}(T x, T y) \leq \varphi(p(x, y)) p(x, T y)+\tau(p(x, y)) p(y, T x) \text { for all } x, y \in X .
$$

Then $\mathcal{F}(T) \neq \emptyset$.

Proof. For each $x \in X$, let $y \in T x$ be arbitrary. Since $p(y, T x)=0$, we have $2 \mathcal{D}_{p}(T x, T y) \leq \varphi(p(x, y)) p(x, T y)$. Therefore, the conclusion follows from Theorem 3.3.

Theorem 3.5. Let $T: X \rightarrow \mathcal{C B}(X)$ be a capable map. Suppose that there exists an $\mathcal{M T}$-function $\phi:[0, \infty) \rightarrow[0,1)$ such that

$$
2 \mathcal{D}_{p}(T x, T y) \leq \varphi(p(x, y))(p(x, T y)+p(y, T x)) \text { for all } x, y \in X .
$$

Then $\mathcal{F}(T) \neq \emptyset$.

Proof. Let $\tau=\phi$. Then, the conclusion follows from Theorem 3.4.

Theorem 3.6. Let $T: X \rightarrow X$ be a selfmap. Suppose that there exists an $\mathcal{M T}$-function $\phi:[0, \infty) \rightarrow[0,1)$ such that

$$
2 d(T x, T y) \leq \varphi(d(x, y))(d(x, T y)+d(y, T x)) \quad \text { for all } x, y \in X .
$$

Then, $T$ has a unique fixed point in $X$.

Proof. Let $p \equiv d$. Then, (3.11) and (3.10) are identical. We prove that $T$ is a capable map. In fact, it suffices to show that (D5) holds. Assume that there exists $w \in X$ with $w \neq T w$ and $\inf \{d(x, w)+d(x, T x): x \in X\}=0$. Then, there exists a sequence $\left\{x_{n}\right\}$ in $X$ such that $\lim _{n \rightarrow \infty}\left(d\left(x_{n}, w\right)+d\left(x_{n}, T x_{n}\right)\right)=0$. It follows that $d\left(x_{n}, w\right) \rightarrow 0$ and $d\left(x_{n}, T x_{n}\right)$ $\rightarrow 0$ and hence $d\left(w, T x_{n}\right) \rightarrow 0$ or $T x_{n} \rightarrow w$ as $n \rightarrow \infty$. By hypothesis, we have

$$
2 d\left(T x_{n}, T w\right) \leq \varphi\left(d\left(x_{n}, w\right)\right)\left(\left(d\left(x_{n}, T w\right)+d\left(w, T x_{n}\right)\right)\right.
$$

for all $n \in \mathbb{N}$. Letting $n \rightarrow \infty$ in (3.12), since $\phi$ is an $\mathcal{M T}$-function and $d\left(x_{n}, w\right) \rightarrow 0$, we have $d(w, T w)<d(w, T w)$, which is a contradiction. So (D5) holds and hence $T$ is a capable map. Applying Theorem 3.5, $\mathcal{F}(T) \neq \emptyset$. Suppose that there exists $u, v \in \mathcal{F}(T)$ with $u \neq v$. Then, by (3.11), we have

$$
2 d(u, v)=2 d(T u, T v) \leq \varphi(d(u, v))((d(u, T v)+d(v, T u))<2 d(u, v),
$$

a contradiction. Hence, $\mathcal{F}(T)$ is a singleton set.

Applying Theorem 3.6, we obtain the following primitive Chatterjea's fixed point theorem [3].

Corollary 3.1. [3] Let $T: X \rightarrow X$ be a selfmap. Suppose that there exists $\gamma \in\left[0, \frac{1}{2}\right)$ such that

$$
d(T x, T y) \leq \gamma(d(x, T y)+d(y, T x)) \text { for all } x, y \in X .
$$

Then, $T$ has a unique fixed point in $X$.

Proof. Define $\phi:[0, \infty) \rightarrow[0,1)$ by $\phi(t)=2 \gamma$. Then, $\phi$ is an $\mathcal{M T}$-function. So (3.13) implies (3.11), and the conclusion is immediate from Theorem 3.6.

Corollary 3.2. Let $T: X \rightarrow \mathcal{C B}(X)$ be a capable map. Suppose that there exist $\alpha, \beta \in\left[0, \frac{1}{2}\right)$ such that 


$$
\mathcal{D}_{p}(T x, T y) \leq \alpha p(x, T y)+\beta p(y, T x) \text { for all } x, y \in X .
$$

Then $\mathcal{F}(T) \neq \emptyset$.

Proof. Let $\phi, \tau:[0, \infty) \rightarrow[0,1)$ be defined by $\phi(t)=2 \alpha$ and $\tau(t)=2 \beta$ for all $t \in[0$, $\infty)$. Then, $\phi$ and $\tau$ are $\mathcal{M T}$-functions, and the conclusion follows from Theorem 3.4.

The following conclusion is immediate from Corollary 3.2 with $\alpha=\beta=\gamma$.

Corollary 3.3. Let $T: X \rightarrow \mathcal{C B}(X)$ be a capable map. Suppose that there exists $\gamma \in\left[0, \frac{1}{2}\right)$ such that

$$
\mathcal{D}_{p}(T x, T y) \leq \gamma(p(x, T y)+p(y, T x)) \quad \text { for all } x, y \in X .
$$

Then $\mathcal{F}(T) \neq \emptyset$.

\section{Remark 3.1.}

(a) Corollary 3.2 and Corollary 3.3 are equivalent. Indeed, it suffices to prove that Corollary 3.2 implies Corollary 3.3. Suppose all assumptions of Corollary 3.2 are satisfied. Let $\gamma:=\max \{\alpha, \beta\}$. Then $\gamma \in\left[0, \frac{1}{2}\right)$ and (3.14) implies (3.15), and the conclusion of Corollary 3.3 follows from Corollary 3.2.

(b) Theorems 3.1-3.4 and Corollaries 3.1 and 3.2 all generalize and improve [5, Theorem 3.4] and the primitive Chatterjea's fixed point theorem [3].

\section{Fixed point theorems of generalized Kannan's type and others}

The following result is given essentially in [5, Theorem 2.1].

Theorem 4.1. Let $T: X \rightarrow \mathcal{C B}(X)$ be a capable map. Suppose that there exists an $\mathcal{M T}$-function $\phi:[0, \infty) \rightarrow[0,1)$ such that for each $x \in X$,

$$
p(y, T y) \leq \varphi(p(x, y)) p(x, y) \text { for all } y \in T x .
$$

Then $\mathcal{F}(T) \neq \emptyset$.

Applying Theorem 4.1, we establish the following new fixed point theorem.

Theorem 4.2. Let $T: X \rightarrow \mathcal{C B}(X)$ be a capable map. Suppose that there exist two $\mathcal{M T}$-functions $\phi, \tau:[0, \infty) \rightarrow[0,1)$ such that for each $x \in X$,

$$
2 \mathcal{D}_{p}(T x, T y) \leq \varphi(p(x, y)) p(x, T x)+\tau(p(x, y)) p(y, T y) \text { for all } y \in T x,
$$

Then $\mathcal{F}(T) \neq \emptyset$.

Proof. Notice that for each $x \in X$, if $y \in T x$, then (4.2) implies

$$
2 p(y, T y) \leq 2 \mathcal{D}_{p}(T x, T y) \leq \varphi(p(x, y)) p(x, T x)+\tau(p(x, y)) p(y, T y)
$$

and hence

$$
p(y, T y) \leq \frac{\varphi(p(x, y))}{2-\tau(p(x, y))} p(x, T x) \leq \varphi(p(x, y)) p(x, y) .
$$

Applying Theorem 4.1, we can get the thesis.

The following conclusion is immediate from Theorem 4.2.

Theorem 4.3. Let $T: X \rightarrow \mathcal{C B}(X)$ be a capable map. Suppose that there exist two $\mathcal{M T}$-functions $\phi, \tau:[0, \infty) \rightarrow[0,1)$ such that 
$2 \mathcal{D}_{p}(T x, T y) \leq \varphi(p(x, y)) p(x, T x)+\tau(p(x, y)) p(y, T y) \quad$ for all $x, y \in X$

Then $\mathcal{F}(T) \neq \emptyset$.

Theorem 4.4. Let $T: X \rightarrow \mathcal{C B}(X)$ be a capable map. Suppose that there exists an $\mathcal{M T}$-function $\phi:[0, \infty) \rightarrow[0,1)$ such that for each $x \in X$,

$2 \mathcal{D}_{p}(T x, T y) \leq \varphi(p(x, y))(p(x, T x)+p(y, T y))$ for all $y \in T x$.

Then $\mathcal{F}(T) \neq \emptyset$.

Theorem 4.5. Let $T: X \rightarrow \mathcal{C B}(X)$ be a capable map. Suppose that there exists an $\mathcal{M T}$-function $\phi:[0, \infty) \rightarrow[0,1)$ such that

$$
2 \mathcal{D}_{p}(T x, T y) \leq \varphi(p(x, y))(p(x, T x)+p(y, T y)) \quad \text { for all } x, y \in X .
$$

Then $\mathcal{F}(T) \neq \emptyset$.

Theorem 4.6. Let $T: X \rightarrow X$ be a selfmap. Suppose that there exists an $\mathcal{M T}$-function $\phi:[0, \infty) \rightarrow[0,1)$ such that

$$
2 d(T x, T y) \leq \varphi(d(x, y))(d(x, T x)+d(y, T y)) \quad \text { for all } x, y \in X
$$

Then, $T$ has a unique fixed point in $X$.

Proof. Let $p \equiv d$. Then, (4.3) and (4.4) are identical. We prove that $T$ is a capable map. In fact, it suffices to show that (D5) holds. Assume that there exists $w \in X$ with $w \neq T w$ and $\inf \{d(x, w)+d(x, T x): x \in X\}=0$. Then, there exists a sequence $\left\{x_{n}\right\}$ in $X$ such that $\lim _{n \rightarrow \infty}\left(d\left(x_{n}, w\right)+d\left(x_{n}, T x_{n}\right)\right)=0$. It follows that $d\left(x_{n}, w\right) \rightarrow 0$ and $d\left(x_{n}, T x_{n}\right)$ $\rightarrow 0$ and hence $d\left(w, T x_{n}\right) \rightarrow 0$ or $T x_{n} \rightarrow w$ as $n \rightarrow \infty$. By hypothesis, we have

$$
2 d\left(T x_{n}, T w\right) \leq \varphi\left(d\left(x_{n}, w\right)\right)\left(\left(d\left(x_{n}, T x_{n}\right)+d(w, T w)\right)\right.
$$

for all $n \in \mathbb{N}$. Since $d\left(x_{n}, w\right) \rightarrow 0$ as $n \rightarrow \infty$ and $\phi$ is an $\mathcal{M T}$-function, $\lim _{n \rightarrow \infty} \phi(d$ $\left.\left(x_{n}, w\right)\right)<1$. Letting $n \rightarrow \infty$ in (4.5), since $T x_{n} \rightarrow w$ and $d\left(x_{n}, T x_{n}\right) \rightarrow 0$ as $n \rightarrow \infty$, we have $2 d(w, T w)<d(w, T w)$, which is a contradiction. So (D5) holds and hence $T$ is a capable map. Applying Theorem $4.5, \mathcal{F}(T) \neq \emptyset$. Suppose that there exists $u, v \in \mathcal{F}(T)$ with $u \neq v$. Then, by (4.4), we have

$$
0<2 d(u, v)=2 d(T u, T v) \leq \varphi(d(u, v))((d(u, T u)+d(v, T v))=0,
$$

a contradiction. Hence, $\mathcal{F}(T)$ is a singleton set.

Applying Theorem 4.6, we obtain the primitive Kannan's fixed point theorem [2].

Corollary 4.1. Let $T: X \rightarrow X$ be a selfmap. Suppose that there exists $\gamma \in\left[0, \frac{1}{2}\right)$ such that

$$
d(T x, T y) \leq \gamma(d(x, T x)+d(y, T y)) \text { for all } x, y \in X
$$

Then $\mathcal{F}(T) \neq \emptyset$.

Corollary 4.2. Let $T: X \rightarrow X$ be a selfmap. Suppose that there exist $\alpha, \beta \in\left[0, \frac{1}{2}\right)$ such that

$$
d(T x, T y) \leq \alpha d(x, T x)+\beta d(y, T y) \text { for all } x, y \in X
$$

Then $\mathcal{F}(T) \neq \varnothing$.

Remark 4.1. Corollary 4.1 and Corollary 4.2 are indeed equivalent. 
Corollary 4.3. Let $T: X \rightarrow \mathcal{C B}(X)$ be a capable map. Suppose that there exist $\alpha, \beta \in\left[0, \frac{1}{2}\right)$ such that

$$
\mathcal{D}_{p}(T x, T y) \leq \alpha p(x, T x)+\beta p(y, T y) \text { for all } x, y \in X .
$$

Then $\mathcal{F}(T) \neq \emptyset$.

Corollary 4.4. Let $T: X \rightarrow \mathcal{C B}(X)$ be a capable map. Suppose that there exists $\gamma \in\left[0, \frac{1}{2}\right)$ such that

$$
\mathcal{D}_{p}(T x, T y) \leq \gamma(p(x, T x)+p(y, T y)) \quad \text { for all } x, y \in X .
$$

Then $\mathcal{F}(T) \neq \emptyset$.

\section{Remark 4.2.}

(a) Corollary 4.3 and Corollary 4.4 are indeed equivalent.

(b) Theorems 4.1-4.6 and Corollaries 4.1-4.4 all generalize and improve [5, Theorem 2.6] and the primitive Kannan's fixed point theorem [2].

\section{Acknowledgements}

The first author was supported by the Natural Science Foundation of Yunnan Province (2010ZC152) and the Scientific Research Foundation from Yunnan Province Education Committee (08Y0338); the second author was supported partially by grant no. NSC 100-2115-M-017-001 of the National Science Council of the Republic of China.

\footnotetext{
Author details

${ }^{1}$ Department of Mathematics, Honghe University, Yunnan 661100, China ${ }^{2}$ Department of Mathematics, National Kaohsiung Normal University, Kaohsiung 824, Taiwan
}

\section{Authors' contributions}

All authors contributed equally and significantly in writing this paper. All authors read and approved the final manuscript.

\section{Competing interests}

The authors declare that they have no competing interests.

Received: 13 August 2011 Accepted: 23 November 2011 Published: 23 November 2011

\section{References}

1. Takahashi, W: Nonlinear Functional Analysis. Yokohama Publishers, Yokohama, Japan (2000)

2. Kannan, R: Some results on fixed point-II. Am Math Mon. 76, 405-408 (1969). doi:10.2307/2316437

3. Shioji, N, Suzuki, T, Takahashi, W: Contractive mappings, Kannan mappings and metric completeness. Proc Am Math Soc. 126, 3117-3124 (1998). doi:10.1090/S0002-9939-98-04605-X

4. Chatterjea, SK: Fixed-point theorems. C R Acad Bulgare Sci. 25, 727-730 (1972)

5. Du, WS: Some new results and generalizations in metric fixed point theory. Nonlinear Anal. 73, 1439-1446 (2010). doi:10.1016/j.na.2010.05.007

6. Du, WS: Coupled fixed point theorems for nonlinear contractions satisfied Mizoguchi-Takahashi's condition in quasiordered metric spaces. Fixed Point Theory and Applications 2010, 9 (2010). Article ID 876372

7. Du, WS: New cone fixed point theorems for nonlinear multivalued maps with their applications. Appl Math Lett. 24 172-178 (2011). doi:10.1016/j.aml.2010.08.040

8. Du, WS: On coincidence point and fixed point theorems for nonlinear multivalued maps. Topology and its Applications. 159, 49-56 (2012). doi:10.1016/.jtopol.2011.07.021

9. Du, WS, Zheng, SX: Nonlinear conditions for coincidence point and fixed point theorems. Taiwan J Math. (in press)

10. Du, WS: Nonlinear contractive conditions for coupled cone fixed point theorems. Fixed Point Theory and Applications 2010, 16 (2010). Article ID 190606

11. Berinde, M, Berinde, V: On a general class of multi-valued weakly Picard mappings. J Math Anal Appl. 326, 772-782 (2007). doi:10.1016/j.jmaa.2006.03.016

12. Mizoguchi, N, Takahashi, W: Fixed point theorems for multivalued mappings on complete metric spaces. J Math Anal Appl. 141, 177-188 (1989). doi:10.1016/0022-247X(89)90214-X

13. Reich, S: Some problems and results in fixed point theory. Contemp Math. 21, 179-187 (1983)

14. Jachymski, J: On Reich's question concerning fixed points of multimaps. Boll Unione Mat Ital. 9(7):453-460 (1995)

15. Suzuki, T: Mizoguchi-Takahashi's fixed point theorem is a real generalization of Nadler's. J Math Anal Appl. 340, 752-755 (2008). doi:10.1016/j.jmaa.2007.08.022 
16. Kada, O, Suzuki, T, Takahashi, W: Nonconvex minimization theorems and fixed point theorems in complete metric spaces. Math Japon. 44, 381-391 (1996)

17. Lin, LJ, Du, WS: Some equivalent formulations of generalized Ekeland's variational principle and their applications. Nonlinear Anal. 67, 187-199 (2007). doi:10.1016/j.na.2006.05.006

18. $\mathrm{Du}, \mathrm{WS}, \mathrm{He}, \mathrm{Z}, \mathrm{Chen}, \mathrm{YL}$ : New existence theorems for approximate coincidence point property and approximate fixed point property with applications to metric fixed point theory, accepted for publication in Journal of Nonlinear and Convex Analysis.

19. Lin, LJ, Du, WS: Ekeland's variational principle, minimax theorems and existence of noncon-vex equilibria in complete metric spaces. J Math Anal Appl. 323, 360-370 (2006). doi:10.1016/j.jmaa.2005.10.005

20. Lin, LJ, Du, WS: On maximal element theorems, variants of Ekeland's variational principle and their applications. Nonlinear Anal. 68, 1246-1262 (2008). doi:10.1016/j.na.2006.12.018

21. Du, WS: Fixed point theorems for generalized Hausdorff metrics. Int Math Forum. 3, 1011-1022 (2008)

22. Du, WS: Critical point theorems for nonlinear dynamical systems and their applications. Fixed Point Theory and Applications2010, 16. Article ID 246382

23. Aubin, JP, Cellina, A: Differential Inclusions. Springer, Berlin (1994)

24. Ding, XP, He, YR: Fixed point theorems for metrically weakly inward set-valued mappings. J Appl Anal. 5(2):283-293 (1999). doi:10.1515/JAA.1999.283

25. Downing, D, Kirk, WA: Fixed point theorems for set-valued mappings in metric and Banach spaces. Math Japon. 22 99-112 (1977)

doi:10.1186/1687-1812-2011-84

Cite this article as: He et al: The existence of fixed points for new nonlinear multivalued maps and their applications. Fixed Point Theory and Applications 2011 2011:84.

\section{Submit your manuscript to a SpringerOpen ${ }^{\odot}$} journal and benefit from:

- Convenient online submission

- Rigorous peer review

- Immediate publication on acceptance

- Open access: articles freely available online

- High visibility within the field

- Retaining the copyright to your article

Submit your next manuscript at $\boldsymbol{s p r i n g e r o p e n . c o m ~}$ 